\title{
The Simulation of Shape Evolution of Solder Joints during Reflow Process and Its Experimental Validation
}

\author{
Ya-Yun Chou ${ }^{1}$, Hung-Ju Chang ${ }^{1}$, Jer-Haur Kuo ${ }^{1}$ and Weng-Sing Hwang ${ }^{1,2}$ \\ ${ }^{1}$ Department of Materials Science and Engineering, National Cheng Kung University, Tainan, Taiwan, R. O. China \\ ${ }^{2}$ Metal Industries Research and Development Centre, Kaohsiung, Taiwan, R. O. China
}

\begin{abstract}
A three-dimensional computer aided analysis system has been developed in this study based on fluid dynamics to simulate the shape evolution of solder joints during the reflow process.

The solution of velocity and pressure fields is based on SOLA (SOLution Algorithm) scheme, and the method to construct the interface and the transportation of volume fractions of liquid in the cells are coupled with PLIC (Piecewise Linear Interface Calculation) and VOF (Volume of Fluid) technologies. In order to consider the effect of surface tension on a fluid surface, the CSF (Continuum Surface Force) model is employed.

The simulated results are compared with experimental measurements and good consistency is observed. Furthermore, the simulated results can reveal the evolution of the molten solder from its initial state to the equilibrium state. It is also capable of analyzing the overflow conditions when the amount of solder deposit is too much to be constrained in the solder pad, which is not achievable by the energy-based technique such as Surface Evolver.
\end{abstract}

(Received November 18, 2005; Accepted February 6, 2006; Published April 15, 2006)

Keywords: mathematical modeling, solder joint, reflow process, Surface Evolver

\section{Introduction}

As electronic packaging technologies continue to move toward smaller scale and higher density, the use of area array type packages have gradually replaced the peripheral type packages. Furthermore, the use of solder joints reduces problems happened in higher frequency, which have been critical issues in the use of wire bonding. Solder joints are used to provide both mechanical and electrical connections for different applications such as flip-chip, ball grid array, chip-scale packaging, and wafer-level packaging. Several works have demonstrated that geometric parameters, such as solder pad size, standoff height, contact angle and etc., significantly influence the fatigue life of the solder joint during thermo/mechanical loading. ${ }^{1,2)}$ Therefore, it is imperative to develop an algorithm to predict the solder joint geometry during reflow process.

Various methods for predicting the reflow shape of solder joints have been developed. In general, the truncated sphere method, the force-balanced analytical solution, and the energy-based method are three major methods in use. ${ }^{3)}$ Among these methods, the energy-based algorithm has the most precise simulation results while packaging is with loading, in which gravity could not be neglected. A robust program Surface Evolver developed by Brakke ${ }^{4,5)}$ is one of algorithms using the energy-based method, and has been successfully applied to deal with problems in electronic packaging processes. However, the restriction of Surface Evolver is that the molten solder does not flow out of the solder pad. Therefore, it is impossible to simulate by Surface Evolver when the volume of solder is too much to be constrained in the solder pad. In order to analyze the overflow condition of the molten solder, and to consider the effect of the solder mask, an algorithm based on fluid dynamics is developed in this study.

\section{Mathematical Method}

\subsection{Description of the physical system}

During the reflow process, molten solders does not flow onto the nonwettable solder mask and is constrained in solder pads, which are usually located at position several micrometers lower than the solder mask. The contact angle of the molten solder not only depends on the surface tension of the molten solder, but also depends on the wall adhesion of the substrate underneath the molten solder. In order to simulate the solder reflow shape in situation which is more approximate to the actual physical system, the surface tension of the molten solder and the wall adhesion between the solder and the substrate have to be considered. Furthermore, the position of the solder pad is usually set at the position lower than the solder mask under the real situation, thus the restriction force of the solder mask because of the gap should be presented. Moreover, owing to the attempt on simulating the shape evolution of solder joints during reflow process, the mathematical model should be able to deal with the transient flow with free surfaces, and to track the location of interface between the vapor and the fluid phases. In this study, the solution of velocity and pressure fields was based on SOLA (SOLution Algorithm) scheme, and free surfaces were tracked using the volume-of-fluid (VOF) method, ${ }^{6)}$ and the surface tension and wall adhesion effects were imposed using the continuum surface force (CSF) model. In order to simplify the simulation system, the change of viscosity of the molten solder with temperature was neglected.

\subsection{Governing equations}

Since the change of viscosity of the molten solder with temperature is neglected, the liquid solder can be approximated as a Newtonian fluid. Moreover, the assumption that the liquid solder is incompressible is imposed for such fluids with a constant density. According to the fluid dynamics theorem, the flow field of an incompressible, Newtonian fluid is governed by Navier-Stokes equations and the continuity 
equation, which represent the conservation of momentum and the conservation of mass respectively.

The momentum equation can be written as:

$$
\begin{aligned}
\frac{\partial \vec{V}}{\partial t}+(\vec{V} \cdot \nabla) \vec{V}= & -\frac{1}{\rho} \nabla p+\frac{\mu}{\rho} \nabla \cdot\left(\nabla \vec{V}+\nabla \vec{V}^{T}\right) \\
& +\vec{g}+\frac{1}{\rho} \vec{F}_{b}
\end{aligned}
$$

where $\vec{V}$ is the velocity field, $\rho$ is density, $P$ is pressure field, $\mu$ is viscosity, $\vec{g}$ is the gravity acceleration, $\vec{F}_{b}$ is the body force. The continuity equation can be written as:

$$
D=\nabla \cdot \vec{V}=0
$$

where $D$ is the divergence.

\subsection{Treatments for the free surface flow}

In order to simulate a transient flow with free surface, the location of free surfaces has to be estimated firstly. Then the change of free surfaces with time is calculated, therefore the evolution of the fluid domain is computed. In this study, the VOF method which was developed by Hirt and Nichols ${ }^{6,7)}$ was adopted to present the fluid domain and to track the evolution of its free surfaces.

In VOF method, free surfaces are represented with discrete volume-of-fluid data on the mesh, which is a scalar field $F(x, y, z, t)$. The field variable $F$ is assigned to each computational element to indicate the volume fraction of fluid in it. Then, free surfaces are reconstructed by means of the volume fraction value $F$, where $F$ is equal to 1 means the cell is full of fluid, $F$ is equal to 0 means the cell is full of gas. While $F$ is between 0 and 1 means the cell contains both fluid and gas, and an interface between fluid and gas is then allocated in this cell. $F$-value is a step function in order to maintain a sharply defined surface. From the law of mass conservation, $F$-value is governed by the following equation:

$$
\frac{\partial F}{\partial t}+(\vec{V} \cdot \nabla) F=0
$$

where $F(x, y, z, 0)$ is known. After the velocity field $\vec{V}$ is computed, $F$-value can be calculated from the above equation. Therefore, free surfaces can be reconstructed from the renewed $F$-value, and volume fluxes can be computed. Finally, new configuration and distribution of fluid is known.

The surface reconstruction method plays an important role in VOF method, which has a great effect on accuracy. A more accurate interface approximation leads to a smoother volume fraction distribution, hence a more precise calculation of surface normal is achieved. In earlier works, the simple line interface calculation (SLIC) method was generally used, which approximates the interface with a straight line aligned with one of the mesh coordinates. However, the SLIC method is a comparatively rougher approximation and is suitable in problems with less deformation involved. The piecewise linear interface calculation (PLIC) method which was developed by Youngs, ${ }^{8}$ allows a non-zero slope for the straight line to approximate the interface, and yields a more accurate interface approximation. These two methods are shown in Fig. 1, where Figs. 1(a) and (b) reconstruct different surface geometries from the same volume fraction data.
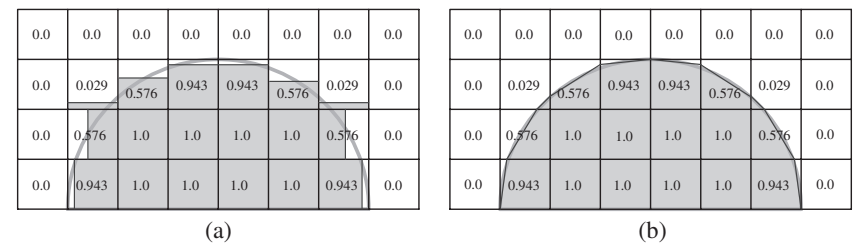

Fig. 1 Two difference methods of reconstructing a surface from the same volume fraction data, where the semicircular curve indicates the actual interface: (a) SLIC approximation, (b) PLIC approximation. ${ }^{9)}$

In this study, the PLIC method coupled with the VOF method were adopted to construct the interface and to calculate the transportation of fluid. The numerical scheme and procedures include the following three steps:

\subsubsection{The construction/reconstruction of free surfaces}

After the $F$-value of each cell is determined, the normal vector of the interface can be calculated from the following equation:

$$
\vec{n}=\nabla F
$$

Therefore, the interface of a surface cell can be represented by the following equation for a plane:

$$
-\vec{x} \cdot \hat{n}-\alpha=0
$$

where $\vec{x}$ is the position vector on interfaces, $\hat{n}$ is the unit normal vector of the plane, $\alpha$ is a plane constant. The negative sign indicates that the direction of the unit normal vector points into gas.

\subsubsection{Solve for the velocity field}

Velocity field and pressure field can be solved from (1) and (2). In this study, an explicit finite difference method and SOLA algorithm, which is a method for solving transient flow, were employed. According to SOLA scheme, a pressure adjustment term is derived from (1) and (2) as follows:

$$
\delta p=-\frac{D}{(\partial D / \partial p)}
$$

In the beginning, a tentative velocity field at new time step is calculated from (1) with a prescribed pressure field at old time step. Then, the divergence is obtained from (2). If the divergence is not equal to zero, that means the calculated velocity and pressure fields could not satisfy the conservation of mass, and the pressure adjustment term $\delta p$ is used to adjust the tentative velocity field. The iteration process is continuing until zero divergence is satisfied for all the cells in the interior region.

\subsubsection{Calculate the convection and redistribution of $F$ - values}

After the velocity and pressure fields are calculated at new time step, the convectional fluid volume and the new location of free surface can therefore be computed in order to solve for the redistribution of $F$-values in each cell. The convectional fluid volume in a layer is estimated approximately equal to the velocity of the cell multiplied by the time interval. Therefore, three volume fluxes: $\left(\phi^{+}\right)_{i, j, k},\left(\phi^{-}\right)_{i, j, k}$ and $\left(\phi^{0}\right)_{i, j, k}$ are calculated. $\left(\phi^{+}\right)_{i, j, k}$ and $\left(\phi^{-}\right)_{i, j, k}$ represent the volume fluxes flow from $(i, j, k)$ cell to $(i+1, j, k)$ and $(i-1, j, k)$ cells respectively. $\left(\phi^{0}\right)_{i, j, k}$ is the remained fluid volume in 


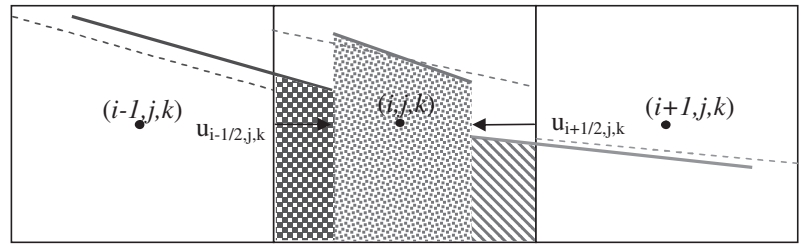

$\left(\phi^{+}\right)_{\mathrm{i}-1, \mathrm{j}, \mathrm{k}} \quad\left(\phi^{0}\right)_{\mathrm{i}, \mathrm{j}, \mathrm{k}} \quad(\phi)_{\mathrm{i}+1, \mathrm{j}, \mathrm{k}}$

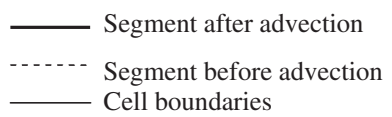

Fig. 2 Calculation of the volume fluxes during one fractional step.

$(i, j, k)$ cell. When the fluid flows out from $(i, j, k)$ cell to $(i+1, j, k)$ cell, $\left(\phi^{+}\right)_{i, j, k}$ is larger than zero and $\left(\phi^{-}\right)_{i+1, j, k}$ is equal to zero. When the fluid flows out from $(i, j, k)$ cell to $(i-1, j, k)$ cell, $\left(\phi^{-}\right)_{i, j, k}$ is larger than zero and $\left(\phi^{+}\right)_{i-1, j, k}$ is equal to zero. The three volume fluxes during one fractional step are regions under the convectional line, which shows in Fig. 2. The redistributed volume fraction in each cell along $x$, $y, z$ directions is then can be given as follows respectively:

$$
\begin{aligned}
& F_{i, j, k}^{(*, x)}=\left(\phi^{+}\right)_{i-1, j, k}+\left(\phi^{0}\right)_{i, j, k}+\left(\phi^{-}\right)_{i+1, j, k} \\
& F_{i, j, k}^{(*, y)}=\left(\phi^{+}\right)_{i, j-1, k}+\left(\phi^{0}\right)_{i, j, k}+\left(\phi^{-}\right)_{i, j+1, k} \\
& F_{i, j, k}^{(*, z)}=\left(\phi^{+}\right)_{i, j, k-1}+\left(\phi^{0}\right)_{i, j, k}+\left(\phi^{-}\right)_{i, j, k+1}
\end{aligned}
$$

where superscript $*$ means the $F$-value at new time step. After $F$-value at new time step in $x, y, z$ axial coordinates are computed from (7), (8) and (9), the redistribution of $F$-values can then be obtained.

\subsection{Treatment of surface tension}

In order to take the effect of surface tension into account, a surface-tension-induced pressure jump was used to represent a boundary condition in the pressure field at the interface in earlier studies. Therefore, the exact surface location and slope had to be computed in order to obtain the correct pressure at the free surface, and a reconstruction of a continuous surface was then required. In this study, a very different approach for reconstructing a continuous surface developed by Brackbill et al. ${ }^{10)}$ was adopted, which is called the CSF model. In the CSF model, surface tension is interpreted as a continuous, three-dimensional effect across an interface, rather than as a boundary condition on the interface. Hence an interfacial surface force is replaced by a localized volume force acting on the interface, which can be added into the Navier-Stokes equations as a form of body force, and has effect on the velocity field.

In CSF model, the interface is regarded as a transition region where the fluid properties change continuously. When the thickness of the transition region is infinitesimal, the volume force $\vec{F}_{b}$ can be written as:

$$
\vec{F}_{b}=\sigma \kappa \hat{n}
$$

where $\sigma$ is the surface tension coefficient, $\hat{n}$ is calculated from VOF data in (4), $\kappa$ is the curvature of free surface, which can be calculated from:

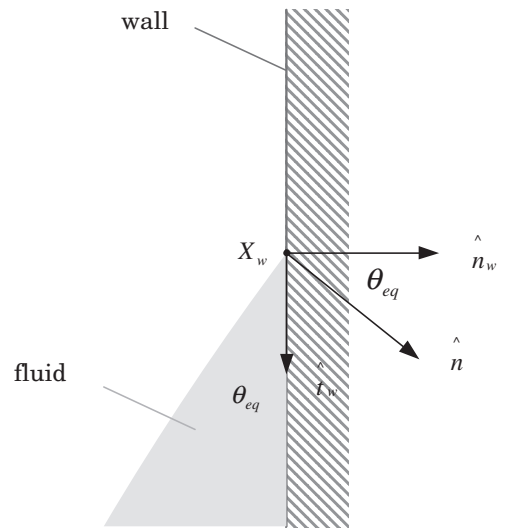

Fig. 3 Magnified view of a static fluid free surface in contact with a wall. ${ }^{11)}$

$$
\kappa=-(\nabla \cdot \hat{n})
$$

This body force $\vec{F}_{b}$ acts everywhere in the transition region, therefore the exact surface location is no longer required. This eliminates the need for interface reconstruction, and simplifies the calculation of surface tension.

\subsection{Treatment of wall adhesion}

When molten solder contacts with the substrate, which includes the wettable solder pad and the nonwettable solder mask, the fluid interface forms a contact angle $\theta$ with the rigid boundary. If the angle $\theta$ is equal to static contact angle $\theta_{e q}$, a state of static equilibrium is reached. If not, a nonzero wall adhesion force results that trends to pull the fluid until $\theta=\theta_{e q} \cdot{ }^{11)}$ This wall adhesion can be regarded as a volume force due to surface tension at points of contact with "walls", therefore, it can be calculated in the same manner as (10), except that a boundary condition is applied to the free surface normal whose vertex lying on or near a rigid boundary. From Fig. 3, the wall adhesion boundary condition can be interpreted as the expression for the unit free surface normal $\hat{n}$ at points $\vec{x}_{w}$ on the wall by geometric identity:

$$
\hat{n}=\hat{n}_{w} \cos \theta_{e q}+\hat{t}_{w} \sin \theta_{e q}
$$

where $\hat{n}_{w}$ is the unit wall normal directed into the wall, $\hat{t}_{w}$ is tangent to the wall and normal to the contact line between the free surface and the wall at $\vec{x}_{w}$.

\section{Experimental Method}

The purpose of experiment conducted in this study is to validate the simulation result of the standoff height and maximum width of solder joints which are related to the change in solder volume. Therefore, solder volume has to be a known value. If the solder bump is made by electroplating, solder volume can not be known easily due to uneven electric current density. Thus, in this study, solder balls were melted together to reform new solder balls with different volume, which was the sum of original volume of each solder balls. The solder ball in use had three different sizes, which was 300,500 and $760 \mu \mathrm{m}$ in diameter respectively, and its composition was 63 mass $\mathrm{Sn}-37$ mass $\mathrm{Pb}$. After solder balls with different volume were made, they were then put onto solder pads with circular shape. These solder pads were 


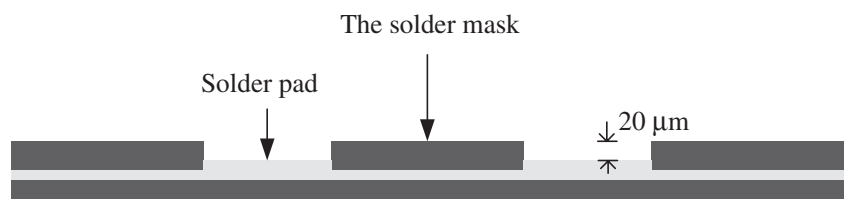

Fig. 4 The cross section of BGA substrate.

$20 \mu \mathrm{m}$ lower than the solder mask, which can be seen in Fig. 4. The material of solder pads was $\mathrm{Ni}-\mathrm{Au}$, and the contact angle of molten solder formed on solder pad was about $5^{\circ}$ by measurement, while the molten solder made an angle about $148.4^{\circ}$ on the epoxy material.

After the volume and contact angle on substrate of solder were known, solder balls put onto solder pads were sent into IR reflow furnace. After the reflow process, the standoff height and maximum width of solder joints were measured and were compared with simulation results.

\section{Results and Discussions}

\subsection{The comparison with Surface Evolver in the simu- lation of contact angle}

The change of contact angle of molten solder on substrate can both be simulated by Surface Evolver and the program developed in this study, and the simulation parameters in use are shown in Table 1 . Contact angle of $30^{\circ}, 50^{\circ}$, and $75^{\circ}$ are taken as examples for the comparison, which can be seen from Fig. 5. Figure 5 shows that there is a good coincidence in simulation results between Surface Evolver and the program developed in this study. In situation where the flow of molten solder on substrate is not restricted, the smaller the contact angle is, the larger the expansion area on substrate is. From the simulation result of the program

Table 1 The simulation parameters used in this study.

(a) In the program developed in this study

\begin{tabular}{cccc}
\hline Composition of solder & Density & Viscosity $^{12)}$ & Surface Tension $^{12)}$ \\
\hline $63 \% \mathrm{Sn}-37 \% \mathrm{~Pb}$ & $8.9 \mathrm{~g} / \mathrm{cm}^{3}$ & $0.002237 \mathrm{~Pa} \cdot \mathrm{s}$ & $49.853 \mathrm{~N} / \mathrm{m}$ \\
\hline (b) In Surface Evolver & & & \\
\hline Composition of solder & Density & Surface Tension \\
\hline $63 \% \mathrm{Sn}-37 \% \mathrm{~Pb}$ & $8.9 \mathrm{~g} / \mathrm{cm}^{3}$ & $49.853 \mathrm{~N} / \mathrm{m}$ \\
\hline
\end{tabular}

Surface Evolver $\quad \underline{\text { Program developed in this study }}$

(a) Contact angle of $30^{\circ}$

(b) Contact angle of $50^{\circ}$

(c) Contact angle of $75^{\circ}$

Fig. 5 The comparison of simulation results in the change of contact angle between Surface Evolver and the program developed in this study. (The vectors represent the surface normal of molten solder in the first layer) developed in this study in Fig. 5, the intensity of adhesive force can be measured by the surface normal of the first layer of molten solder in contact with the substrate. Thus, the smaller the contact angle is, the larger the normal vectors of interfaces are, which represent a greater adhesive force to the substrate.

\subsection{The simulation of shape evolution of solder joints during reflow process}

Since the program developed in this study is based on fluid dynamics, the flow behavior of molten solder during reflow process can be simulated by calculating the change of velocity field at each time step. However, Surface Evolver calculates the shape evolution of solder by conjugate gradient methods, which have no physical significance at every iteration step, especially when the volume of solder is too much to be constrained in the solder pad. Therefore, the understanding of the shape evolution of solder joints during reflow process has to depend on the simulation based on fluid dynamics scheme, such as the program developed in this study. The simulation results are shown in Fig. 6, and the solder volume is set to be $0.2298 \mathrm{~mm}^{3}$.

As can be seen from Fig. 6, if the initial shape of solder is a cylinder, it evolves into sphere gradually with time during reflow process due to the effect of surface tension. In the program developed in this study, the solder mask can be set to be higher than solder pad as the actual situation, which is not achievable by Surface Evolver. Thus the simulation results can be closer to the real situation when the solder mask has great influence on the flow of molten solder. However, it is impossible to take the pictures of molten solder during reflow process in $10^{-6}$ second such a short interval, especially when the temperature in reflow furnace is over $220^{\circ} \mathrm{C}$. Therefore, this simulation result can not easily be validated with experiment.

\subsection{The effect of solder volume with experimental validation}

Considering the effect of solder volume, solder balls of different volume were put onto circular solder pads which are $600 \mu \mathrm{m}$ in diameter. The standoff height and maximum width of solder joints after reflow, was measured and then compared with simulation results. It can be seen from Fig. 7, that simulation results consist with the experimental measurements very well. Therefore, the accuracy of the program developed in this study is verified in an accurate range. On the solder pad of the same size, the standoff height and maximum width of solder joints increase with increasing solder volume. However, simulation results are slightly larger than the experiment measurements, and this may due to the volume shrinkage during the solidification of solder.

The shape of solder joints are observed from the cross sections, which are compared with simulation results as shown in Fig. 8. As can be seen from Fig. 8, the contact angle formed in solder pads increases with the increasing solder volume. It is different from situation in Fig. 5, where the expansion of solder on the substrate is not restricted, and contact angle of solder is irrelevant to the solder volume. In Figure 8 , the flow of solder is confined in solder pads by the 


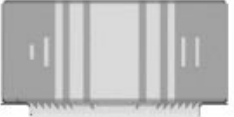

$0 \mu \mathrm{s}$

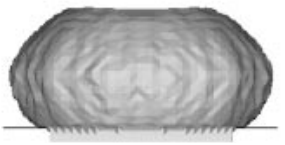

$69 \mu \mathrm{s} \quad \rightarrow$

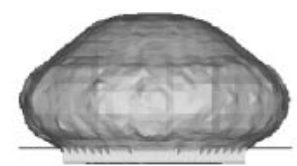

$151 \mu \mathrm{s}$

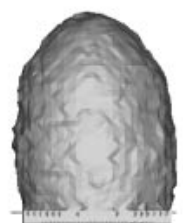

$944 \mu \mathrm{s} \rightarrow$

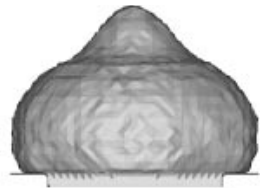

$227 \mu \mathrm{s}$

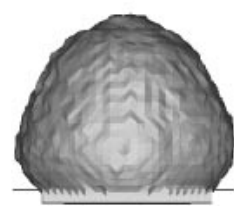

$\rightarrow \quad 446 \mu \mathrm{s} \quad \rightarrow$

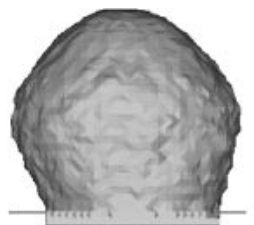

$\rightarrow$

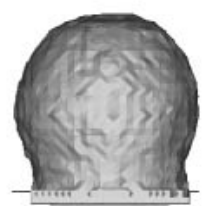

$681 \mu \mathrm{s}$

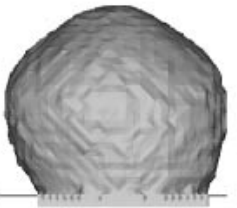

$1440 \mu \mathrm{s}$

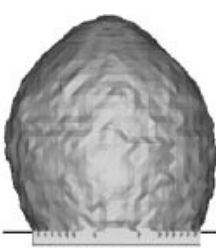

$1265 \mu \mathrm{s}$

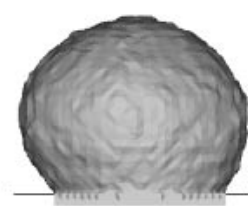

$1508 \mu \mathrm{s}$

Fig. 6 The simulation of shape evolution of solder joints during reflow process.

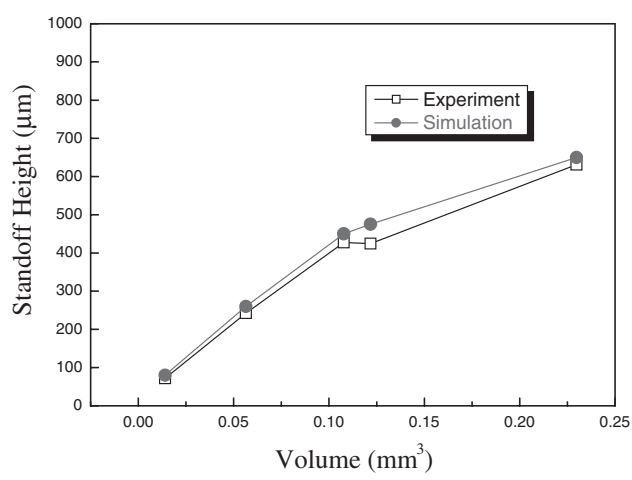

(a) The change in standoff height

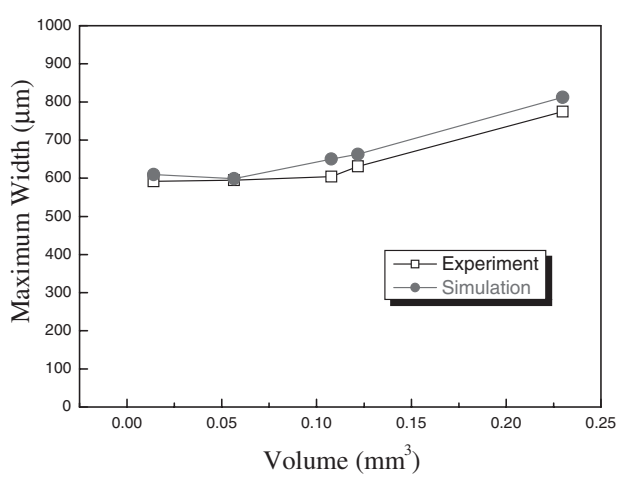

(b) The change in maximum width

Fig. 7 Comparison the simulation results of standoff height and maximum width of solder with experimental measurement.

nonwettable solder mask, which is $20 \mu \mathrm{m}$ higher than solder pads. Therefore, the contact angle of solder is different from the static contact angle value, and is proportional to the solder volume.

\subsection{The effect of size and shape of solder pad}

Solder volume of $0.0141 \mathrm{~mm}^{3}$ were put onto circular solder pads which were 600 and $420 \mu \mathrm{m}$ in diameter respectively. The simulated standoff height and maximum width of solder joints after reflow are listed in Table 2. From Table 2 it can be seen that the solder joint on the solder pad with larger size has the lower standoff height, which due to the larger wettable area.

If the size of solder pad is fixed, the effect of the shape of solder pad is able to be observed. The solder pad area of $0.2827 \mathrm{~mm}^{2}$ was used. Then solder volume of $0.2298 \mathrm{~mm}^{3}$ was put onto the circular and rectangular solder pads respectively. The simulated tandoff height and maximum width of solder after reflow are shown in Table 3. As can be seen from Table 3, there is no much difference in standoff height and maximum width of solder between on circular and rectangular solder pad, except for slightly lower in height and smaller in width of solder on the circular solder pad.

Therefore, the shape of solder joints is observed in Fig. 9. It shows that on circular and rectangular solder pads of equivalent area, the length of rectangular solder pad is slightly larger than the diameter of circular solder pad. Moreover, the distribution of molten solder on the circular solder pad is more uniform than on the rectangular solder pad but not disperse into four corners as on the rectangular pad. This may causes slightly lower in height and smaller in the width of solder joint on the circular solder pad. 
(a) Volume $=0.0141 \mathrm{~mm}^{3}$

(b) Volume $=0.0564 \mathrm{~mm}^{3}$

(c) Volume $=0.1077 \mathrm{~mm}^{3}$

(d) Volume $=0.1218 \mathrm{~mm}^{3}$
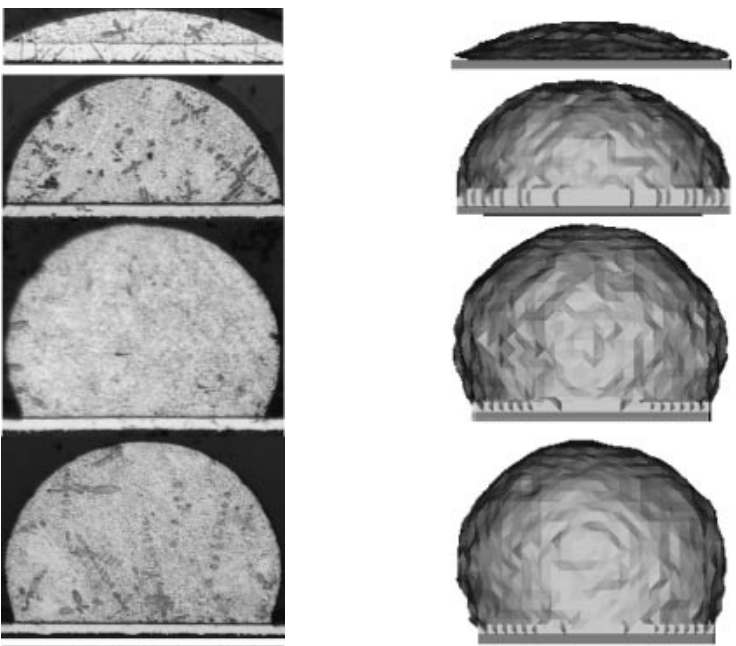

(e) Volume $=0.2298 \mathrm{~mm}^{3}$
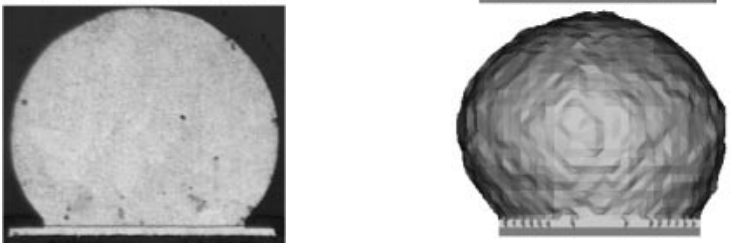

Fig. 8 The experimental observation on cross sections of solder joints of different volume compared with simulation results.

Table 2 The effect of size of solder pads on solder joints.

\begin{tabular}{ccc}
\hline $\begin{array}{c}\text { Diameter of } \\
\text { solder pad }\end{array}$ & $\begin{array}{c}\text { Standoff height } \\
\text { of solder }\end{array}$ & $\begin{array}{c}\text { Maximum width } \\
\text { of solder }\end{array}$ \\
\hline $600 \mu \mathrm{m}$ & $80 \mu \mathrm{m}$ & $591.9 \mu \mathrm{m}$ \\
\hline $420 \mu \mathrm{m}$ & $175 \mu \mathrm{m}$ & $454.1 \mu \mathrm{m}$ \\
\hline
\end{tabular}

Solder volume $=0.0141 \mathrm{~mm}^{3}$

Table 3 The effect of shape of solder pads on solder joints.

\begin{tabular}{ccc}
\hline $\begin{array}{c}\text { The shape of } \\
\text { solder pad }\end{array}$ & $\begin{array}{c}\text { Standoff height } \\
\text { of solder }\end{array}$ & $\begin{array}{c}\text { Maximum width } \\
\text { of solder }\end{array}$ \\
\hline Circular & $650 \mu \mathrm{m}$ & $812.6 \mu \mathrm{m}$ \\
\hline Rectangular & $626.6 \mu \mathrm{m}$ & $823.8 \mu \mathrm{m}$ \\
\hline
\end{tabular}

Solder volume $=0.2298 \mathrm{~mm}^{3}$, solder pad area $=0.2827 \mathrm{~mm}^{2}$

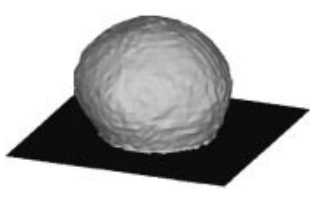

(a) Solder joint on circular solder pad

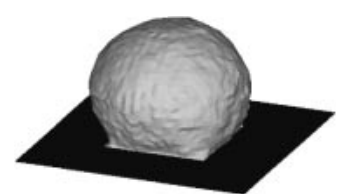

(b) Solder joint on rectangular solder pad

Fig. 9 The comparison of the simulation results in shape between solder joint on circular solder pad and on rectangular solder pad.

\subsection{The effect of wettability of the solder mask}

The contact angle of molten solder on substrate was used to characterize the wettability of substrate in this study. Contact angle of $90^{\circ}$ and $148^{\circ}$ were used to represent solder masks of two materials, where contact angle of $90^{\circ}$ had inferior wettability to molten solder. Solder volume of $0.2298 \mathrm{~mm}^{3}$ was put onto the circular solder pad which was $600 \mu \mathrm{m}$ in diameter, and the solder masks of different

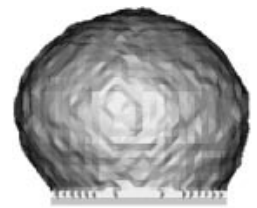

(a) The contact angle of the solder mask is $148^{\circ}$.

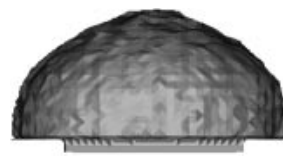

(b) The contact angle of the solder mask is $90^{\circ}$
Fig. 10 The simulation results of considering the effect of the wettability of the solder mask.

wettabilities were compared as shown in Fig. 10. In the same solder volume, when the wettability of the solder mask is inferior, the ability of the solder mask to confine the molten solder in the solder pad is better. Contact angle of $90^{\circ}$ of the solder mask shown in Fig. 10 apparently can not constrain such mass of solder in the solder pad, and causes the solder flow out onto the solder mask, thus a contact angle of $90^{\circ}$ between the solder and the solder mask is formed. Therefore, when the volume of solder is larger, an overflow condition is appeared if the restriction of the solder mask is not enough. In the simulation by Surface Evolver, the wettability of the solder mask is not considered since the molten solder is assumed not flow out onto the solder mask.

\subsection{The effect of the position of the solder mask}

In the same bump pitch, the volume of solder should be larger enough to allow for the assembly, but not too large to cause the contact of adjacent solder joints and make the short in circuit. Therefore, a maximum volume of solder under the given bump pitch is imperative to obtain. In this study, the position of the solder mask was considered to understand its effect on the determination of maximum volume of the solder, which was not able to be considered in Surface 


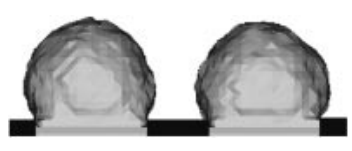

(a) The solder pad is lower than the solder mask

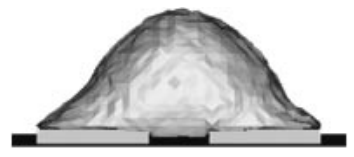

(b) The solder pad is higher than the solder mask
Fig. 11 The simulation results of considering the effect of the position of solder mask.

Evolver. Two cases were compared: the solder pad is lower than the solder mask, and the solder pad is higher than the solder mask. A given volume of solder is the same, one can be seen in Fig. 11, that adjacent solder joints contact with each other in the situation where the solder pad is higher than the solder mask. Thus, under the restriction of the thickness of solder mask, the larger maximum volume of solder is obtained in the same bump pitch.

\section{Conclusions}

In order to understand the shape evolution of solder joints during reflow process, a three-dimensional computer-aided analysis system based on fluid dynamics has been developed in this study. The developed program is capable of simulating the change of contact angle of the solder on the substrate, which is consistent with the simulation results by the energybased algorithm, the Surface Evolver. In addition, the effect of solder volume on the shape of solder joints was simulated and the results were verified to have good coincidence with experimental measurements. Furthermore, the effect of size and the shape of solder pad were simulated and reasonable results were obtained.

On the advantage of using algorithms based on fluid dynamics, the shape evolution of solder joints during reflow process and the overflow condition of the molten solder were able to simulate. Moreover, the physical properties of the solder mask such as wettability and thickness were allowed to consider, which was not achievable by the Surface Evolver.

\section{Acknowledgements}

This work has been supported by the National Science Council in Taiwan (NSC92-2216-E-006-041), for which the authors are grateful.

\section{REFERENCES}

1) H. K. Charles and G. V. Clatterbaugh: ASME Journal of Electronic Packaging 112 (1990) 135-146.

2) M. K. Shah: ASME Journal of Electronic Packaging 118 (1990) 122126.

3) K. N. Chiang and C. A. Yuan: IEEE Transactions on Advanced Packaging 24 (2001) 158-162.

4) K. A. Brakke: Version 2.20, Mathematics Department, Susquehanna University, Selinsgrove, 2003.

5) K. A. Brakke: Experimental Mathematics 1 (1992) 141-165.

6) C. W. Hirt, B. D. Nichols and R. S. Hotchkiss: Technology Report LA8355, (Los Alamos Scientific Laboratory, 1980).

7) C. W. Hirt and B. D. Nichols: J. Comput. Phys. 39 (1981) 201-225.

8) D. L. Youngs: Numerical Methods for Fluid Dynamics, ed. by K. W. Morton and M. J. Baines, (Academic Press, 1982) pp. 273-285.

9) A. Celic and G. G. Zilliac: Computational Study of Surface Tension and Wall Adhesion Effects on an Oil Film Flow Underneath an Air Boundary Layer, (NASA Ames Research Center, USA, August 1997).

10) J. U. Brackbill, D. B. Kothe and C. Zemach: J. Comput. Phys. 100 (1992) 335-354.

11) D. B. Kothe, R. C. Mjolsness and M. D. Torrey: "RIPPLE: A Computer Program for Incompressible Flows with Free Surfaces", LA-12007MS, April 1994.

12) M. Dietzal, S. Haferl, Y. Ventikos and D. Poulikakos: J. Heat Trans. 125 (2003) 365-376. 